\title{
The Role of Mifepristone in Meningiomas Management: A Systematic Review of the Literature
}

\author{
Giulia Cossu, ${ }^{1}$ Marc Levivier, ${ }^{1}$ Roy Thomas Daniel, ${ }^{1}$ and Mahmoud Messerer ${ }^{1,2}$ \\ ${ }^{1}$ Service of Neurosurgery, Department of Clinical Neuroscience, Faculty of Human Medicine and Biology, \\ University Hospital of Lausanne, 46 rue du Bugnon, 1011 Lausanne, Switzerland \\ ${ }^{2}$ Department of Neurosurgery, University Hospital of Bicetre, Faculty of Medicine of Paris Sud, 78 rue du Général Leclerc, \\ 94270 Le Kremlin-Bicêtre, France
}

Correspondence should be addressed to Giulia Cossu; giulia.css@gmail.com

Received 26 February 2015; Accepted 27 April 2015

Academic Editor: Maria Caffo

Copyright (C) 2015 Giulia Cossu et al. This is an open access article distributed under the Creative Commons Attribution License, which permits unrestricted use, distribution, and reproduction in any medium, provided the original work is properly cited.

Objectives. We performed a systematic literature review to analyze the clinical application and the safety of mifepristone, a prominent antiprogesterone agent, in meningioma patients. Materials and Methods. A systematic search was performed through Medline, Cochrane, and clinicaltrials.gov databases from 1960 to 2014. Study Selection. Studies were selected through a PICO approach. Population was meningioma patients, meningioma cells cultures, and animal models. Intervention was mifepristone administration. Control was placebo administration or any other drug tested. Outcomes were clinical and radiological responsiveness, safety profile, and cell growth inhibition. Results. A total of 7 preclinical and 6 clinical studies and one abstract were included. Encouraging results were found in preclinical studies. Concerning clinical studies, the response rate to mifepristone in terms of radiological regression and symptomatic improvement/stability in patients with inoperable meningioma was low. In meningiomatosis, favorable preliminary results were recorded. The safety profile was good. Limitations were as follows. The tumoral expression of progesterone receptors was not analyzed systematically in every study considered. Conclusions. No clear evidence exists to recommend mifepristone in inoperable meningiomas. Preliminary encouraging results were found in diffuse meningiomatosis. Mifepristone is a well-tolerated treatment. Patients' selection and hormonal profile analysis in meningiomas are fundamental for a better understanding of its benefit. Multicenter placebo-controlled trials are required.

\section{Introduction}

Meningiomas represent the most common primary intracranial neoplasm, with an annual incidence of 7-8 cases/100000 individuals [1]. Surgery represents the first line of treatment for symptomatic or growing meningiomas and the recurrence rate is proportional to the extent of resection [2]. Gross total resection may be challenging with lesions located near critical neurovascular structures or highly infiltrating the bone or the dura. Technical advances in microsurgical techniques, endoscopic approaches, and radiation therapies, especially radiosurgery, have recently improved the management of recurrent meningiomas.

Epidemiological studies have reported a higher prevalence of meningioma in females [1] and further studies showed a positive association between meningioma and breast cancer with a twofold increase in risk [3]. Similarly, waxing and waning of clinical manifestations during pregnancy, more evident during the last four months of gestations, were observed $[4,5]$. These observations led to the hypothesis that meningioma growth may be hormonally affected or dependent.

Donnell et al. [6] first described estrogen receptor (ER) expression in meningioma specimens. Further histochemical staining has shown how, contrarily to breast cancer cells, meningioma cells present a high expression of progesterone receptors (PR) and only a weak positivity for ER [7-9]. In most progesterone receptor positive tissues, estrogens modulate the presence of PR [10] but this seems not to be the case for meningiomas. PR were found also in normal leptomeningeal tissue and they may influence the normal functioning of the meninges [11]. 
Many hormonal antagonists have been used for antineoplastic purpose. Mifepristone (RU-486) was a French discovery in 1982 and it has been used as a drug to terminate early pregnancy. It is an oral progesterone antagonist with a minor affinity for the glucocorticoid receptor [12]. Given its different hormonal and endocrine properties, a number of clinical trials started to investigate its alternative applications in the oncologic field [13-21]. The aim of this paper is to perform a systematic review of the literature regarding the oncological use of mifepristone in meningiomas, to clarify its clinical indications and the long-term safety.

\section{Materials and Methods}

The literature search was performed by one of the authors (Giulia Cossu) through the electronic databases Medline and Cochrane from January 1960 till December 2014, using the search terms "mifepristone" and "meningioma," as free text. We also manually reviewed the reference lists of identified studies and scanned abstracts from recent (from 2001 to 2015) conference proceedings. Finally, we searched for ongoing trials on https://clinicaltrials.gov/.

2.1. Study Selection. Two authors (Giulia Cossu and Mahmoud Messerer) reviewed independently full-text articles, abstracts, and citations to select pertinent studies. Search was focused on the usefulness of mifepristone treatment and treatment related side effects in patients with meningiomas: the study selection was based on a PICO approach. Population included patients with a diagnosis of meningioma, both confirmed histologically and suspected by radiological characteristics. Intervention was mifepristone administration and the control was represented by the administration of placebo or another drug. The primary outcomes considered were the radiological response (diminution or stabilization of tumor volume assessed through serial CT or MRI) and the clinical improvement, either subjectively declared from the patients or objectively assessed through a medical examination, evaluated during and/or after treatment administration. The secondary outcomes evaluated were the clinical tolerance and the side effects related to mifepristone treatment. Randomized controlled trials were preferred but also full-text articles of retrospective or prospective studies evaluating the efficacy or the feasibility of mifepristone treatment in meningiomas were included. Preclinical studies, using cell cultures or animal models, were also considered. Considering the paucity of literature data on the topic, we decided to include case reports and case series when satisfying clinical and radiological evaluations were present. Abstract and posters reporting unpublished data were included. Review studies and studies not in English were not further considered.

In case of disagreement about the inclusion of a study, this was discussed and considered eligible only if a consensus from all the examiners was reached.

\section{Results}

With our literature search, 67 articles were identified and screened on the basis of title and abstract. Two abstracts were also considered pertinent to the topic.
A total of 7 preclinical and 6 clinical studies and one abstract were included in our systematic review (Figure 1, flow chart).

3.1. Preclinical Studies (Table 1). In 1986, Olson et al. [22] first demonstrated the efficacy of mifepristone in inhibiting cell growth in three cultures of human meningioma cells after 28 days of treatment. The histological grade was not specified but all the specimens were positive for PR and weakly positive for ER. Mifepristone seemed to compete with progesterone for the progesterone-binding protein site in meningioma cells with an inhibition of cell growth ranging between 18 and $36 \%$. A clear dose effect was seen only in one of three specimens, thus indicating that a competitive binding may not be solely responsible for these results [22].

Blankenstein et al. [23] reported similar results in 30 cultures of human meningioma cells exposed to estrogen, progesterone, tamoxifen, and mifepristone at variable concentrations for 8 days. Cell growth was significantly reduced by mifepristone exposure $(p<0.05)$ when compared to control cultures.

According to Koper et al. [24], progesterone and mifepristone may modulate the sensibility of meningioma cells to the epidermal growth factor (EGF): culture cells treated with mifepristone showed in fact a diminished response to the mitogenic effect of EGF. However, the PR expression was not analyzed in one case and the EGF receptors (EGFR) expression was not specified in 2 cases.

Schrell et al. [25] showed no effect from progesterone or mifepristone exposition in 23 meningioma cell cultures with varying degrees of PR expression. The thymidine-labeled uptake was unaffected by progestogenic treatment and the DNA polymerase activity was not correlated with the PR expression. Analogously, Wilisch-Neumann et al. [26] reported a very limited response from mifepristone treatment: the growth inhibition was evident in one of four analyzed specimens and the concentration of mifepristone needed was too elevated to be used in common clinical practice.

Matsuda et al. [27] showed interesting results: they conducted an in vitro study associated with an in vivo evaluation after implantation of tumoral cells in the subrenal capsule of nude mice. Mifepristone showed a cytostatic and a cytocidal effect independently from PR expression in vitro. In fact, over 9 specimens not expressing PR, 7 responded to treatment. Similar results were found in the animal model, where an inhibition of tumor growth was independent of PR expression in the meningioma cells implanted.

Olson et al. [28] used 6 nude mice to perform a placebocontrolled study. They obtained satisfying results with regression of the volume of the implanted meningioma in two of three cases, and only a small amount of cells was found in the third case receiving mifepristone.

In vitro and preclinical studies supported thus the realization of clinical trials to evaluate the efficacy of mifepristone in patients with unresectable meningioma.

3.2. Clinical Trials in Meningioma Patients (Table 2). All included full-text studies were retrospective. Five studies evaluated the effect of mifepristone therapy on the clinical 


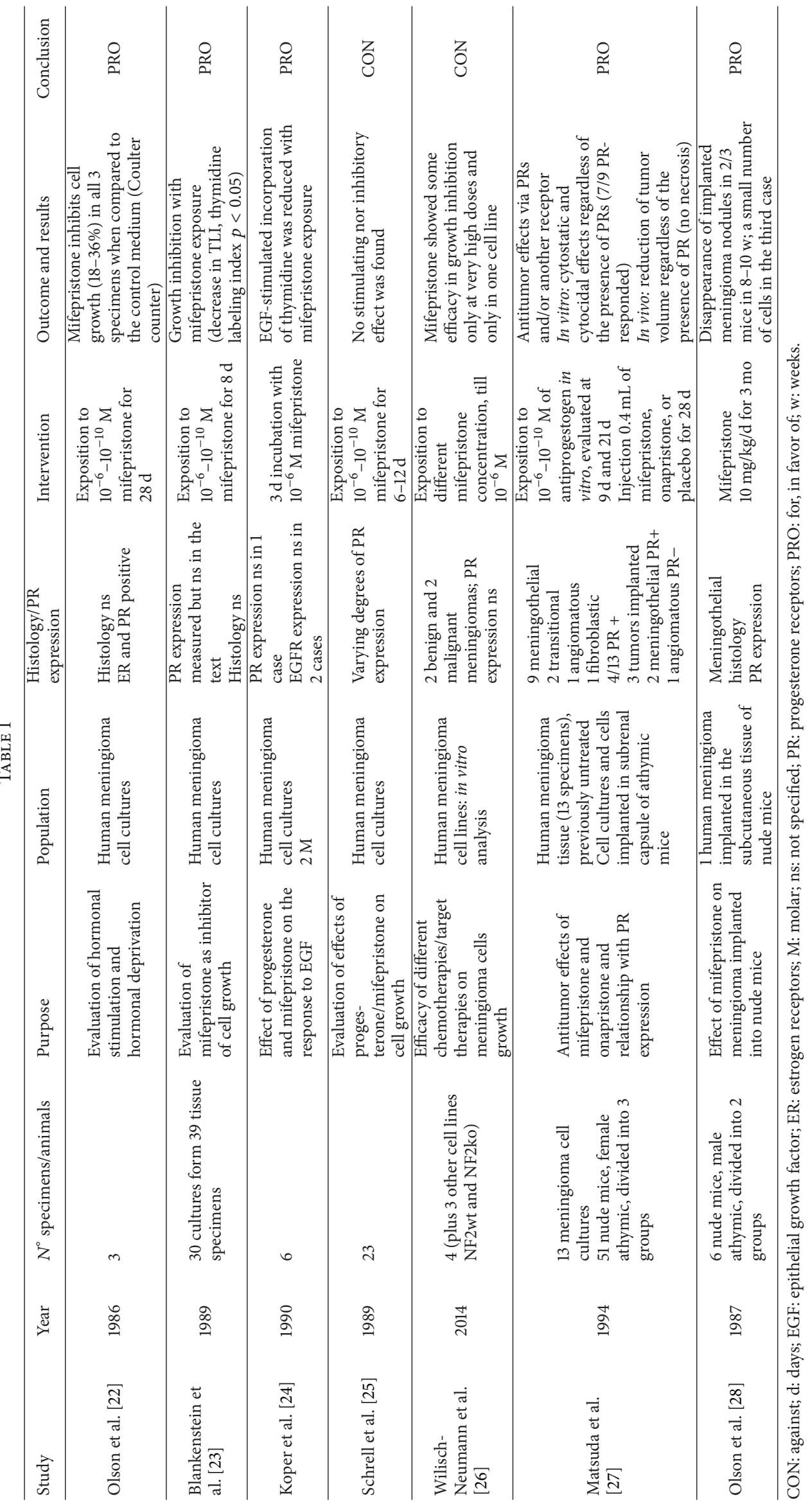




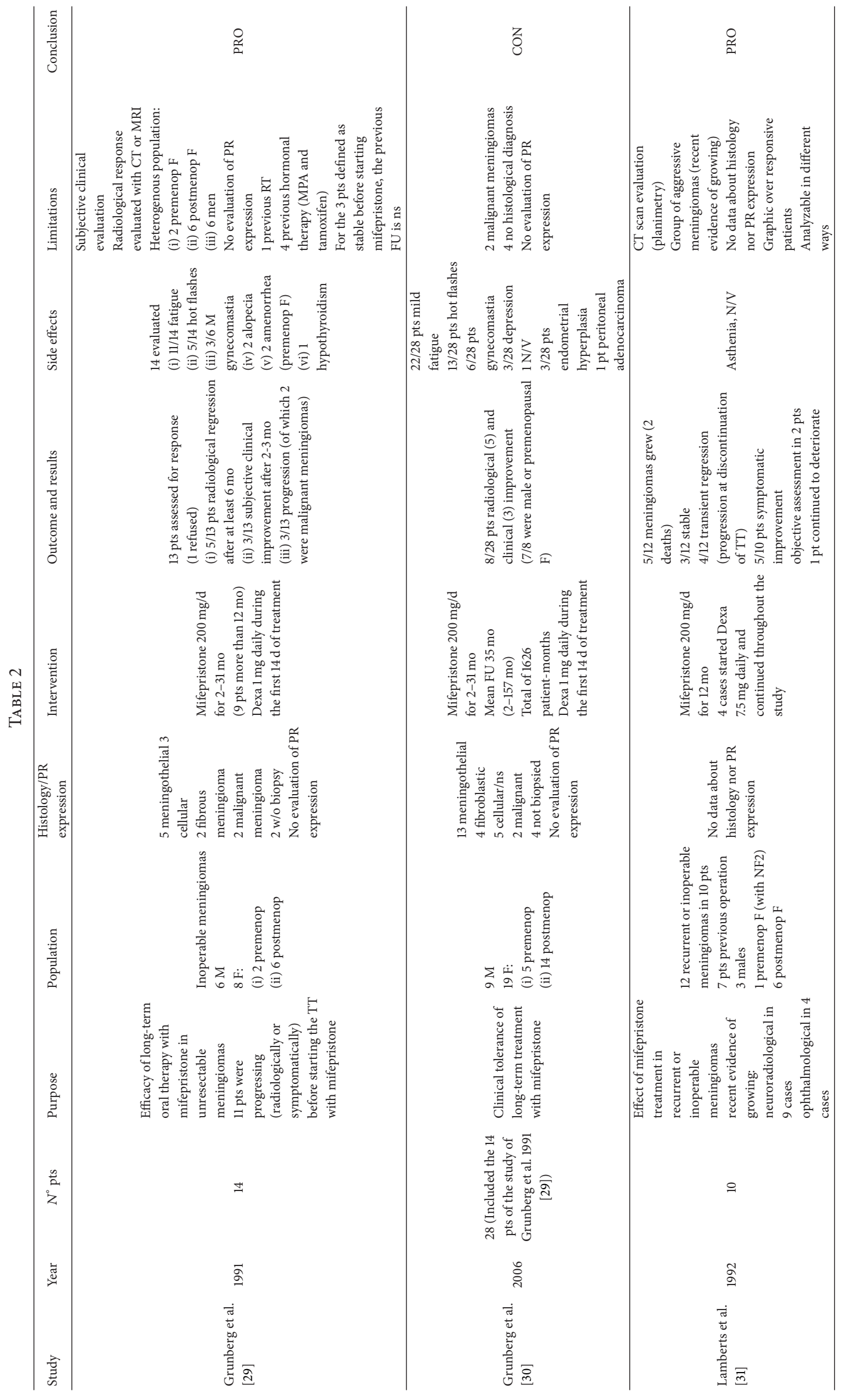




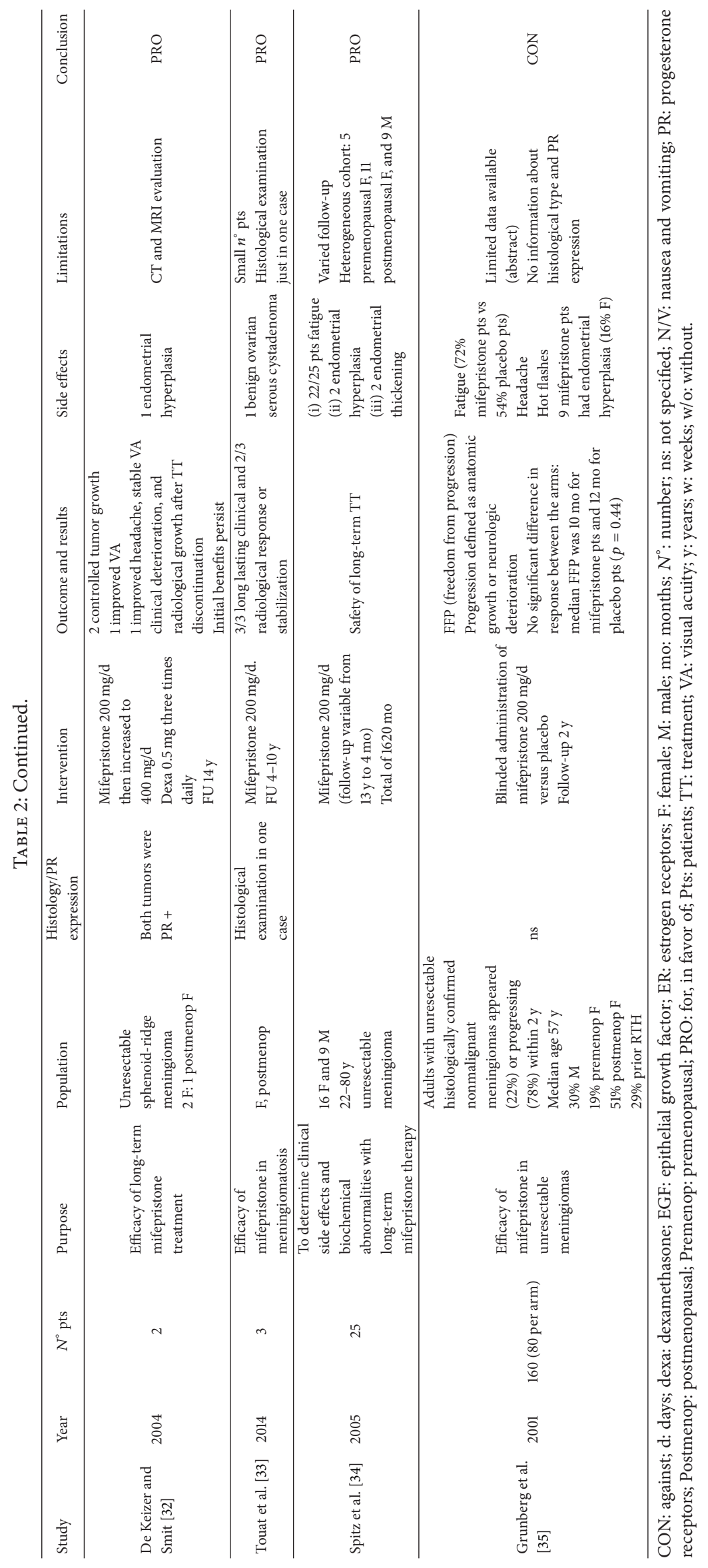




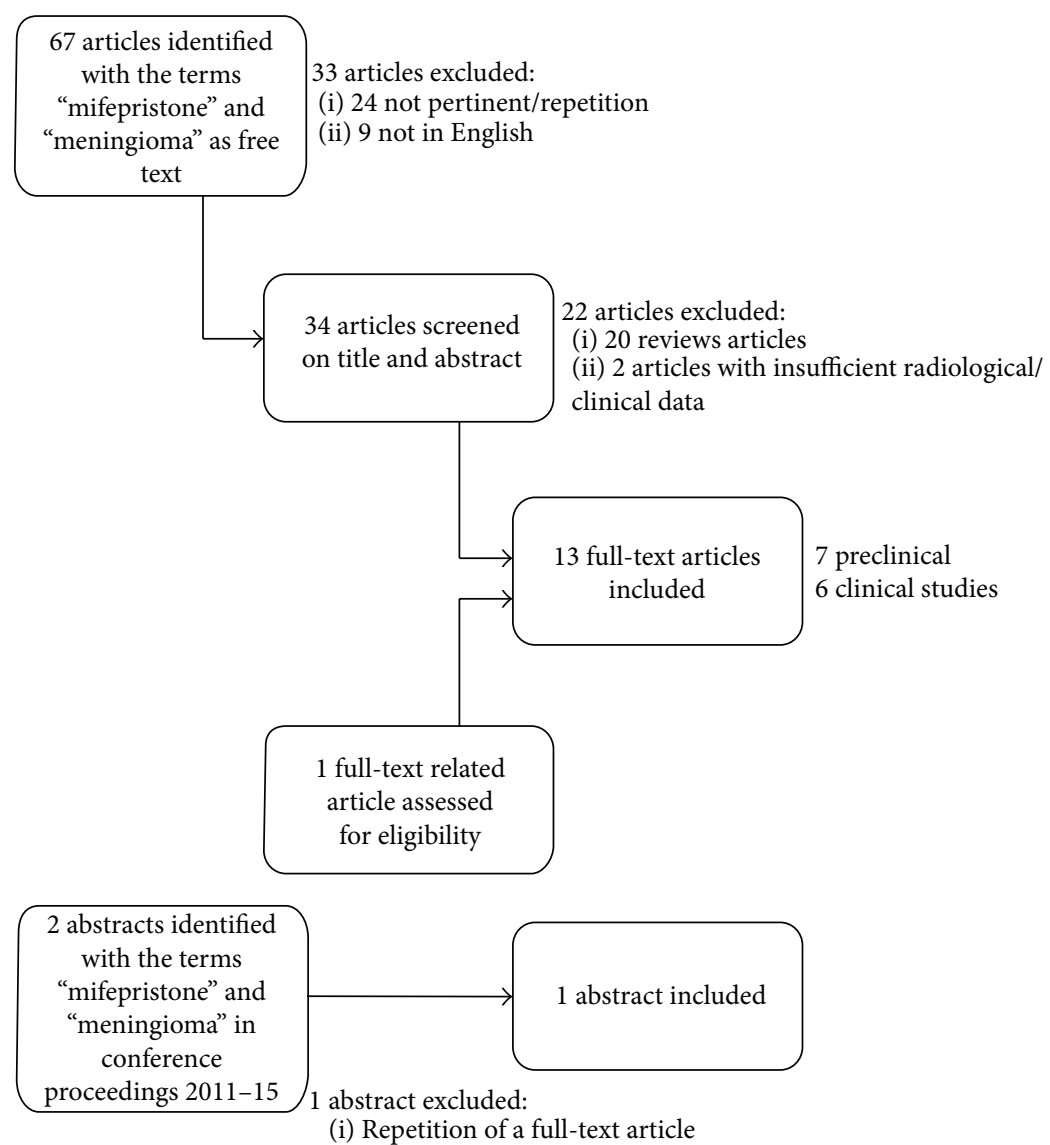

Figure 1: Flow chart.

and radiological follow-up of meningioma patients [29-33]. One study focused on the evaluation of side effects of longterm mifepristone treatment [34]. All the studies considered focused on patients with unresectable or recurrent meningiomas, with progression at clinical or radiological follow-up in most of the cases, with the exception of the study of Touat et al. [33], which was conducted on three patients with diffuse meningiomatosis.

The abstract included reported preliminary results of a phase III double-blind randomized placebo-controlled study conducted in patients with unresectable meningiomas [35].

The dose of $200 \mathrm{mg} /$ day was chosen to obtain a good antiprogesterone effect without a significant antiglucocorticoid activity $[36,37]$. The duration of the treatment varied among the studies from 2 to 31 months, and the follow-up period extended from the duration of the treatment to 14 years. To avoid adrenal insufficiency most authors introduced a substitutive treatment of dexamethasone during the first weeks or during the whole length of the treatment [29-32].

3.2.1. Therapeutic Effects. Grunberg et al. reported preliminary results in 14 patients in 1991 [29]. Thirteen patients were evaluated for the clinical and radiological follow-up and in 5 of the 13 patients (38\%) tumor regression was recorded, accompanied by a subjective symptomatic improvement in 3 of them (23\%). Three patients experienced progression
(23\%) and two of them had malignant meningioma. Daily oral mifepristone for a period of 2 years or more was well tolerated.

Given these encouraging results, the same group started a phase III randomized double-blind placebo-controlled trial on the usefulness of mifepristone treatment on 160 patients with progressive meningiomas. An abstract was presented in 2001 and authors failed to demonstrate improvement in freedom from progression in mifepristone group $(p=$ 0.44) [35]. However hormone receptors expression was not specified. These results were never further published.

Grunberg et al. reported in 2006 more detailed results in 28 patients, including the patients previously enrolled in the study of 1991 [30]. The mean follow-up was 35 months. A radiological improvement with reduction in tumor size was reported in 5 of the 28 patients $(17 \%)$ and a clinical improvement was evident in 3 patients out of 28 (11\%). Seven of 8 patients experimenting regression (both clinical and radiological) were males or premenopausal females.

Lamberts et al. [31] evaluated a cohort of 10 patients with 12 recurrent or inoperable meningiomas, progressing at follow-up. They recorded a transient regression in 33\% of the tumors (4/12) with further radiological progression after the discontinuation of the treatment. The disease was stable in $25 \%$ cases $(3 / 12)$, while $42 \%$ (5/12) progressed. Fifty percent of the patients (5/10) reported clinical improvement, 
objectively assessed in 2 cases. No data about PR expression were provided.

De Keizer and Smit [32] reported a case series of 2 patients treated with long-term mifepristone for unresectable sphenoid-ridge meningioma, with a follow-up of 14 years. Both patients experienced radiological stability and symptomatic improvement: one reported an improvement in visual acuity and the other experienced regression of his headache with no changes in visual acuity. Both patients progressed and deteriorated after treatment discontinuation with a new stabilization after the restarting of the treatment.

Touat et al. [33] enrolled three postmenopausal patients with diffuse meningiomatosis. The histological examination was performed only in one case. All the patients experimented long-lasting clinical remission and in two of them meningiomas stabilized or diminished in size after mifepristone treatment.

3.2.2. Side Effects. The most common described side effect of mifepristone therapy is asthenia. Severe fatigue however is rare. Some patients experienced anorexia, vomiting, and nausea $[29,34]$. All these symptoms may be attributed to the blockade of glucocorticoid receptors and an improvement of these manifestations with an exogenous corticosteroid treatment was described $[29,31]$. The majority of the studies supplemented patients under mifepristone treatment with daily low-dose of dexamethasone, at least for the first two weeks or for the whole duration of the treatment.

Hot flashes, gynecomastia or breast tenderness, decreased libido, and cutaneous rash were also observed [29]. The cessation of menses was an expected side effect in premenopausal patients, with a return to a normal menstrual cycle after a variable period of time after the discontinuation of the treatment [29]. Supplementation in testosterone helped in normalizing libido in male patients [29]. Mifepristone treatment was associated with the development of endometrial hyperplasia [30, 32, 34, 35].

One case of peritoneal adenocarcinoma [30] and one case of benign ovarian serous cystadenoma [33] were reported. The association with mifepristone treatment is unknown.

Subclinical hypothyroidism was observed [38] and some authors reported transient elevation of hepatic enzymes during mifepristone treatment for other conditions $[39,40]$ but this finding was not confirmed by Spitz et al. in meningioma patients [34].

\section{Discussion}

Meningiomas have a significant association with hormonedependent conditions $[3,4,41]$ and since the discovery of the high prevalence of PR expression in meningiomas, the option to use hormonal therapy as cytostatic agent became a real therapeutic strategy.

Mifepristone is a 19-norsteroid agent presenting a high affinity for the PR and a lower but considerable affinity for the glucocorticoid receptor [12]. The abortifacient properties of mifepristone were early discovered and the use of this drug is currently approved for early termination of pregnancy, cervical dilatation for surgical abortion, and management of early embryonic loss [42]. Further applications to the endocrine and oncological fields remain under investigation.

The expression of PR in meningioma cells was early characterized in 1979 [6] and, since then, different hormonal therapies have been used in preclinical studies and clinical trials. Most of them showed contrasting results [43-48], while initial encouraging results were found for mifepristone in in vitro studies and animal models.

Clinical studies on the efficacy of the use of mifepristone in meningiomas showed contrasting results. Grunberg et al. [29] in their study of 1991 reported positive results in 38\% patients with inoperable meningiomas. A further enrollment allowed the same group to publish more extensive data: in 2006, the authors failed to show new cases of radiological or clinical response to mifepristone treatment [30]. Analogously in 2001 they presented in an abstract the results of a placebocontrolled RCT in patients with unresectable meningiomas: no significant difference on freedom from progression was observed between the two arms [35]. Unfortunately, limited data are available for this trial.

Lamberts et al. [31] showed a stable disease or a transient regression in tumor volume in $58 \%$ of cases ( $7 / 12$ meningiomas), but when the time course of tumor size (volumetric data) was analyzed in more details, the results were no more convincing. The four tumors decreasing in size showed a progressive reduction of tumor volume only in one case during mifepristone treatment, while in two cases a transient precocious regression was followed by a dimensional increase during mifepristone treatment. In one case, an initial stability was followed by a slight reduction of tumor volume at one year of treatment.

De Keizer and Smit [32] showed a radiological stability and a symptomatic improvement in both patients studied, having an unresectable sphenoid-ridge meningioma.

Two case reports, not included in the primary analysis because of the lacking of precise radiological data, confirmed a radiological and clinical stability with long-term mifepristone treatment in two patients $[49,50]$ and a decrease in tumor size in one case [50].

Long-term treatment with mifepristone is generally well tolerated. Adrenal insufficiency may be seen with very high doses of mifepristone, where the compensatory elevation of endogenous cortisol becomes insufficient, but in most of cases the introduction of a low-dose therapy of daily dexamethasone per os was sufficient to relieve the mild symptoms of corticotropic insufficiency (asthenia, nausea, and vomiting) [30,31].

Mifepristone treatment was associated with the development of endometrial hyperplasia $[30,32,34]$ and endometrial polyps [49]. The hormonal pathways determining this phenomenon are still unknown. Heikinheimoab et al. [38] investigated the effects of long-term mifepristone treatment ( $200 \mathrm{mg} / \mathrm{d}$ for at least 15 months) on sex steroids and gonadotrophins levels. A prolonged treatment is associated with increased serum levels of androstenedione, testosterone, estrone, and estradiol, probably through the inhibition of glucocorticoid receptors. An increase in cortisol level secondary to mifepristone administration may be in fact associated 
with an increase in adrenal production of androgens with a secondary aromatization to estrogens $[38,51,52]$. The proliferative effects of estrogens are in these cases no more counteracted by progesterone.

The case of subclinical hypothyroidism recorded [38] was probably linked to an interaction with the corticotropic axis $[53,54]$ or due to an effect on synthesis and/or release of thyroxine (biologically characterized by an elevation of thyroid-stimulating hormone and a decrease in thyroxin) rather than a direct interaction of mifepristone with the thyroxine receptor [55].

In conclusion, mifepristone seems to act on the progesterone, glucocorticoid, androgen, and thyroid pathways on the long term.

From our literature analysis only retrospective studies were identified and included as full-text articles. One RCT was only available as abstract. Thus, no class I evidence may be extrapolated. Also two case series, respectively, of 2 [32] and 3 patients [33] were included. The experience in clinical practice is very limited and the major evidence derives from one single center $[29,30,35]$.

The studies were extremely heterogeneous both in the preclinical and in the clinical groups, in terms of population included, duration of treatment, and length of follow-up. For all these reasons, a meta-analysis was not performed.

In clinical studies, a heterogeneous cohort of patients in terms of sex, menopausal status, histology, and expression of PR was enrolled and data were pooled together in the majority of cases. Furthermore, some authors included cases with no histologic diagnosis $[29,30]$ and the specific expression of PR was not analyzed in most of cases [29-31, 33].

The selection of the population of interest may be determinant: Grunberg et al. [30] observed significant responses in males and in premenopausal women, and further studies focusing on this population may help in detecting clinically significant responses deriving form mifepristone treatment.

The histology and the WHO grade may be important in determining the response to mifepristone treatment. A lower presence of PR was noted in anaplastic meningiomas [56] and PR expression was inversely correlated with Ki67 expression and WHO grading $[57,58]$. Thus, mifepristone may not be a good treatment for anaplastic and malignant meningiomas.

Encouraging results support the use of mifepristone in a clinical trial for patients with diffuse meningiomatosis. Touat et al. [33] enrolled three patients and all showed a long-lasting clinical improvement during the treatment, associated with two cases with a radiological shrinking or stabilization. From epidemiological studies, it is evident that diffuse meningiomatosis is characterized by a more pronounced female predominance and immunohistochemical studies showed a higher PR expression in this subgroup of meningiomas [59].

Progesterone receptors exist as two isoforms (PRA and PRB) codified from the same gene by different promoters [60]. Increasing literature evidence shows how they have different functions $[61,62]$ and PRA seems to be downregulated in models of antiprogestin resistant breast cancers $[63,64]$. Mifepristone showed a major action in preclinical models of breast cancers overexpressing PRA when compared to cancers having a high PRB expression [65]. These effects may be useful in guiding the choice of target patients and it would be interesting to analyze more in details the relationship between the PR isoforms expressed and the responsiveness to mifepristone treatment in meningioma patients.

Some authors support the hypothesis that the antitumoral effect of mifepristone may not depend directly on the expression of PR [66]. Koper et al. [24] showed how progesterone may act in vitro through the modulation of the cellular response to various mitogenic stimuli as EGF, insulin, and insulin-like growth factor 1 (IGF-1). Mifepristone inhibits growth induced by EGF in vitro [24] and it may block the activation of IGF-1 signaling in some breast cancer cell lines [67]. These may be additional mechanisms of action of this drug. Furthermore, mifepristone inhibited cell migration, abnormal adhesion to basement membrane, and angiogenesis in an animal model of adenocarcinoma [68]. Thereafter, it was shown to promote apoptosis through the activation of the pathway of caspase-3 [18]. Further investigations should be conducted to study the interactions between mifepristone and its modulations of signal transduction pathways.

Further limitations of the studies included in this review are related to the volumetric assessment of tumor size, which was not recorded systematically by all the authors, and the radiological techniques varied between CT scan and MRI, even in the same studies. Indeed, a well-performed MRI is more sensible in detecting minimal changes in size than a simple CT evaluation. Also, the length of the treatment and the follow-up period should be sufficiently long to detect minimal volumetric changes; the effects of a hormonal therapy may in fact be evident after a certain period of time, because of the slow proliferation rate of meningioma cells. Furthermore, clinical improvement should be objectively documented when possible (periodical neurological examination of cranial nerve palsies, ophthalmological evaluation for visual acuity and visual fields, etc.).

Through the analysis of the literature, only minor evidences exist to recommend mifepristone treatment in surgically inoperable progressing meningiomas. This hormonal therapy has a safe profile and a good clinical tolerance, but the clinical benefit is very limited and it should be recommended only in an experimental setting. The indications for diffuse meningiomatosis are still preliminary. Well-designed multicentered placebo-controlled trails with a long followup should be realized to finally determine the real value of mifepristone treatment.

Mifepristone may represent a starting point towards new perspectives: other hormonal therapies and in particular other progesterone antagonists with higher affinity for PR may represent a real future alternative. Furthermore advances in molecular and genomic studies are actually targeting the molecular changes in tumorigenic mechanisms to obtain more selective therapies. Inhibitors of EGFR or PDGFR may inhibit meningioma growth but preliminary studies failed to show a clinical benefit $[69,70]$. Less selective tyrosine kinase receptor inhibitors, like sunitinib, may be more promising $[70,71]$. Also antiangiogenetic molecules may represent a valuable option [72]. 


\section{Conclusions}

The recurrence rate is high for meningiomas incompletely resected and different therapeutic options were explored for the management of recurrent or inoperable meningiomas. The characterization of hormonal receptors, in particular PR, opened new ways towards antiprogestogens as therapeutic strategies.

According to literature analysis no clear evidence exists to recommend mifepristone treatment in recurrent and inoperable meningiomas. A possible application may be found in diffuse meningiomatosis but further confirmations are needed. Mifepristone is a well-tolerated treatment; however a preliminary selection of the patients is fundamental.

It is time the clinical experience available from single center studies is substituted by well-designed multicenter placebo-controlled studies.

\section{Conflict of Interests}

On behalf of all authors, the corresponding author states that there is no conflict of interests.

\section{References}

[1] Q. T. Ostrom, H. Gittleman, P. Liao et al., "CBTRUS statistical report: primary brain and central nervous system tumors diagnosed in the United States in 2007-2011," Neuro-Oncology, vol. 16, supplement 4, pp. ivl-iv63, 2014.

[2] D. Simpson, "The recurrence of intracranial meningiomas after surgical treatment," Journal of Neurology, Neurosurgery, and Psychiatry, vol. 20, no. 1, pp. 22-39, 1957.

[3] B. S. Schoenberg, B. W. Christine, and J. P. Whisnant, "Nervous system neoplasms and primary malignancies of other sites. The unique association between meningiomas and breast cancer," Neurology, vol. 25, no. 8, pp. 705-712, 1975.

[4] E. R. Bickerstaff, J. M. Small, and I. A. Guest, "The relapsing course of certain meningiomas in relation to pregnancy and menstruation," Journal of Neurology, Neurosurgery \& Psychiatry, vol. 21, no. 2, pp. 89-91, 1958.

[5] R. D. Weyand, C. C. Mac, and R. B. Wilson, "The effect of pregnancy on intracranial meningiomas occurring about the optic chiasm," The Surgical Clinics of North America, vol. 31, no. 4, pp. 1225-1233, 1951.

[6] M. S. Donnell, G. A. Meyer, and W. L. Donegan, "Estrogenreceptor protein in intracranial meningiomas," Journal of Neurosurgery, vol. 50, no. 4, pp. 499-502, 1979.

[7] M. A. Blankenstein, G. Blaauw, S. W. J. Lamberts, and E. Mulder, "Presence of progesterone receptors and absence of oestrogen receptors in human intracranial meningioma cytosols," European Journal of Cancer \& Clinical Oncology, vol. 19, no. 3, pp. 365-370, 1983.

[8] M. A. Blankenstein, C. van der Meulen-Dijk, and J. H. H. Thijssen, "Assay of oestrogen and progestin receptors in human meningioma cytosols using immunological methods," Clinica Chimica Acta, vol. 165, no. 2-3, pp. 189-195, 1987.

[9] C. Bozzetti, R. Camisa, R. Nizzoli et al., "Estrogen and progesterone receptors in human meningiomas: biochemical and immunocytochemical evaluation," Surgical Neurology, vol. 43, no. 3, pp. 230-234, 1995.
[10] W. L. McGuire, K. B. Horwitz, O. H. Pearson, and A. Segaloff, "Current status of estrogen and progesterone receptors in breast cancer," Cancer, vol. 39, no. 6, pp. 2934-2947, 1977.

[11] M. Poisson, H. Magdelenat, P. M. Martin, and et al, "Progestin receptors in human normal leptomeninges in adults," Revue Neurologique, vol. 140, no. 1, pp. 14-19, 1984.

[12] E.-E. Baulieu, "Contragestion and other clinical applications of RU 486, an antiprogesterone at the receptor," Science, vol. 245, no. 4924, pp. 1351-1357, 1989.

[13] R. Fjelldal, B. T. Moe, A. Ørbo, and G. Sager, "MCF-7 cell apoptosis and cell cycle arrest: non-genomic effects of progesterone and mifepristone (RU-486)," Anticancer Research, vol. 30, no. 12, pp. 4835-4840, 2010.

[14] C. R. Tieszen, A. A. Goyeneche, B. N. Brandhagen, C. T. Ortbahn, and C. M. Telleria, "Antiprogestin mifepristone inhibits the growth of cancer cells of reproductive and non-reproductive origin regardless of progesterone receptor expression," $B M C$ Cancer, vol. 11, article 207, 2011.

[15] M. F. El Etreby, Y. Liang, and R. W. Lewis, "Induction of apoptosis by mifepristone and tamoxifen in human LNCaP prostate cancer cells in culture," The Prostate, vol. 43, no. 1, pp. 31-42, 2000.

[16] M.-F. Lin, M. H. Kawachi, M. R. Stallcup, S. M. Grunberg, and F.-F. Lin, "Growth inhibition of androgen-insensitive human prostate carcinoma cells by a 19-norsteroid derivative agent, mifepristone," The Prostate, vol. 26, no. 4, pp. 194-204, 1995.

[17] A. A. Goyeneche, R. W. Carón, and C. M. Telleria, "Mifepristone inhibits ovarian cancer cell growth in vitro and in vivo," Clinical Cancer Research, vol. 13, no. 11, pp. 3370-3379, 2007.

[18] D.-Q. Li, Z.-B. Wang, J. Bai et al., "Reversal of multidrug resistance in drug-resistant human gastric cancer cell line SGC7901/VCR by antiprogestin drug mifepristone," World Journal of Gastroenterology, vol. 10, no. 12, pp. 1722-1725, 2004.

[19] Q.-L. Sun, X.-G. Zhang, Q.-T. Xing et al., "A study of mifepristone/IFN- $\gamma$-induced apoptosis of human cholangiocarcinoma cell line FRH-0201 in vitro," OncoTargets and Therapy, vol. 5, pp. 335-342, 2012.

[20] M. A. Navo, J. A. Smith, A. Gaikwad, T. Burke, J. Brown, and L. M. Ramondetta, "In vitro evaluation of the growth inhibition and apoptosis effect of mifepristone (RU486) in human Ishikawa and HEC1A endometrial cancer cell lines," Cancer Chemotherapy and Pharmacology, vol. 62, no. 3, pp. 483489, 2008.

[21] R. Jurado, A. Lopez-Flores, A. Alvarez, and P. García-López, "Cisplatin cytotoxicity is increased by mifepristone in cervical carcinoma: an in vitro and in vivo study," Oncology Reports, vol. 22, no. 5, pp. 1237-1245, 2009.

[22] J. J. Olson, D. W. Beck, J. Schlechte, and P.-M. Loh, "Hormonal manipulation of meningiomas in vitro," Journal of Neurosurgery, vol. 65, no. 1, pp. 99-107, 1986.

[23] M. A. Blankenstein, C. van der Meulen-Dijk, and J. H. H. Thijssen, "Effect of steroids and antisteroids on human meningioma cells in primary culture," Journal of Steroid Biochemistry, vol. 34, no. 1-6, pp. 419-421, 1989.

[24] J. W. Koper, J. A. Foekens, R. Braakman, and S. W. J. Lamberts, "Effects of progesterone on the response to epidermal growth factor and other growth factors in cultured human meningioma cells," Cancer Research, vol. 50, no. 9, pp. 2604-2607, 1990.

[25] U. M. H. Schrell, E. F. Adams, R. Fahlbusch et al., "Hormone dependency of meningiomas," The Lancet, vol. 333, no. 8651, pp. 1381-1382, 1989. 
[26] A. Wilisch-Neumann, D. Pachow, M. Wallesch et al., "Reevaluation of cytostatic therapies for meningiomas in vitro," Journal of Cancer Research and Clinical Oncology, vol. 140, no. 8, pp. 1343-1352, 2014.

[27] Y. Matsuda, K. Kawamoto, K. Kiya, K. Kurisu, K. Sugiyama, and T. Uozumi, "Antitumor effects of antiprogesterones on human meningioma cells in vitro and in vivo," Journal of Neurosurgery, vol. 80, no. 3, pp. 527-534, 1994.

[28] J. J. Olson, D. W. Beck, J. A. Schlechte, and P.-M. Loh, "Effect of the antiprogesterone RU-38486 on meningioma implanted into nude mice," Journal of Neurosurgery, vol. 66, no. 4, pp. 584-587, 1987.

[29] S. M. Grunberg, M. H. Weiss, I. M. Spitz et al., "Treatment of unresectable meningiomas with the antiprogesterone agent mifepristone," Journal of Neurosurgery, vol. 74, no. 6, pp. 861866, 1991.

[30] S. M. Grunberg, M. H. Weiss, C. A. Russell et al., "Long-term administration of mifepristone (RU486): clinical tolerance during extended treatment of meningioma," Cancer Investigation, vol. 24, no. 8, pp. 727-733, 2006.

[31] S. W. J. Lamberts, H. L. J. Tanghe, C. J. J. Avezaat et al., "Mifepristone (RU 486) treatment of meningiomas," Journal of Neurology Neurosurgery and Psychiatry, vol. 55, no. 6, pp. 486490, 1992.

[32] R. J. W. de Keizer and J. W. A. Smit, "Mifepristone treatment in patients with surgically incurable sphenoid-ridge meningioma: a long-term follow-up," Eye, vol. 18, no. 9, pp. 954-958, 2004.

[33] M. Touat, G. Lombardi, P. Farina, M. Kalamarides, and M. Sanson, "Successful treatment of multiple intracranial meningiomas with the antiprogesterone receptor agent mifepristone (RU486)," Acta Neurochirurgica, vol. 156, no. 10, pp. 1831-1835, 2014.

[34] I. M. Spitz, S. M. Grunberg, N. Chabbert-Buffet, T. Lindenberg, H. Gelber, and R. Sitruk-Ware, "Management of patients receiving long-term treatment with mifepristone," Fertility and Sterility, vol. 84, no. 6, pp. 1719-1726, 2005.

[35] S. M. Grunberg, C. Rankin, J. Townsend, J. Ahmadi, L. Feun, and R. Fredericks, Eds., Phase III Double-Blind Randomized Placebo-Controlled Study on Mifepristone (RU) for the Treatment of Unresectable Meningioma, American Society of Clinical Oncology, Alexandria, Va, USA, 2001.

[36] L. K. Nieman, T. M. Choate, G. P. Chrousos et al., "The progesterone antagonist RU 486. A potential new contraceptive agent," The New England Journal of Medicine, vol. 316, no. 4, pp. 187-191, 1987.

[37] B. Couzinet, N. Le Strat, A. Ulmann, E. E. Baulieu, and G. Schaison, "Termination of early pregnancy by the progesterone antagonist RU 486 (Mifepristone)," The New England Journal of Medicine, vol. 315, no. 25, pp. 1565-1570, 1986.

[38] O. Heikinheimoab, S. Ranta, S. Grunberg, and I. M. Spitz, "Alterations in sex steroids and gonadotropins in postmenopausal women subsequent to long-term mifepristone administration," Steroids, vol. 65, no. 10-11, pp. 831-836, 2000.

[39] A. A. Murphy, A. J. Morales, L. M. Kettel, and S. S. C. Yen, "Regression of uterine leiomyomata to the antiprogesterone RU486: dose-response effect," Fertility and Sterility, vol. 64, no. 1, pp. 187-190, 1995.

[40] S. H. Eisinger, S. Meldrum, K. Fiscella, H. D. le Roux, and D. S. Guzick, "Low-dose mifepristone for uterine leiomyomata," Obstetrics and Gynecology, vol. 101, no. 2, pp. 243-250, 2003.
[41] N. C. A. Roelvink, W. Kamphorst, H. A. M. van Alphen, and B. R. Rao, "Pregnancy-related primary brain and spinal tumors," Archives of Neurology, vol. 44, no. 2, pp. 209-215, 1987.

[42] R. Sitruk-Ware and I. M. Spitz, "Pharmacological properties of mifepristone: toxicology and safety in animal and human studies," Contraception, vol. 68, no. 6, pp. 409-420, 2003.

[43] J. W. Goodwin, J. Crowley, H. J. Eyre, B. Stafford, K. A. Jaeckle, and J. J. Townsend, "A phase II evaluation of tamoxifen in unresectable or refractory meningiomas: a Southwest Oncology Group study, Journal of Neuro-Oncology, vol. 15, no. 1, pp. 7577, 1993.

[44] S. M. Grunberg and M. H. Weiss, "Lack of efficacy of megestrol acetate in the treatment of unresectable meningioma," Journal of Neuro-Oncology, vol. 8, no. 1, pp. 61-65, 1990.

[45] J. Jääskeläinen, E. Laasonen, J. Kärkkäinen, M. Haltia, and H. Troupp, "Hormone treatment of meningiomas: lack of response to medroxyprogesterone acetate (MPA) - a pilot study of five cases," Acta Neurochirurgica, vol. 80, no. 1-2, pp. 35-41, 1986.

[46] J. R. Jay, D. T. MacLaughlin, K. R. Riley, and R. L. Martuza, "Modulation of meningioma cell growth by sex steroid hormones in vitro," Journal of Neurosurgery, vol. 62, no. 5, pp. 757762, 1985.

[47] E. R. Waelti and T.-M. Markwalder, "Endocrine manipulation of meningiomas with medroxyprogesterone acetate: effect of MPA on growth of primary meningioma cells in monolayer tissue culture," Surgical Neurology, vol. 31, no. 2, pp. 96-100, 1989.

[48] T.-M. Markwalder, H. A. Gerber, E. Waelti, T. Schaffner, and R. V. Markwalder, "Hormonotherapy of meningiomas with medroxyprogesterone acetate. Immunohistochemical demonstration of the effect of medroxyprogesterone acetate on growth fractions of meningioma cells using the monoclonal antibody Ki-67," Surgical Neurology, vol. 30, no. 2, pp. 97-101, 1988.

[49] P. A. Martineau and M. Levental, "Large endometrial polyp in a patient on long-term mifepristone therapy," Journal of Ultrasound in Medicine, vol. 19, no. 7, pp. 487-489, 2000.

[50] H. R. Haak, R. J. W. de Keizer, J. C. W. Hagenouw-Taal, A. P. van Seters, G. J. Vielvoye, and H. van Dulken, "Successful mifepristone treatment of recurrent, inoperable meningioma," The Lancet, vol. 336, no. 8707, pp. 124-125, 1990.

[51] S. W. J. Lamberts, J. W. Koper, and F. H. de Jong, "The endocrine effects of long-term treatment with mifepristone (RU 486)," The Journal of Clinical Endocrinology \& Metabolism, vol. 73, no. 1, pp. 187-191, 1991.

[52] O. Heikinheimo, S. Ranta, S. Grunberg, P. Lähteenmäki, and I. M. Spitz, "Alterations in the pituitary-thyroid and pituitaryadrenal axes-consequences of long-term mifepristone treatment," Metabolism: Clinical and Experimental, vol. 46, no. 3, pp. 292-296, 1997.

[53] Y. Takiyama, H. Tanaka, Y. Takiyama, and I. Makino, "The effects of hydrocortisone and RU486 (mifepristone) on iodide uptake in porcine thyroid cells in primary culture," Endocrinology, vol. 135, no. 5, pp. 1972-1979, 1994.

[54] D. Rubello, N. Sonino, D. Casara, M. E. Girelli, B. Busnardo, and M. Boscaro, "Acute and chronic effects of high glucocorticoid levels on hypothalamic-pituitary-thyroid axis in man," Journal of Endocrinological Investigation, vol. 15, no. 6, pp. 437-441, 1992.

[55] S. M. Grunberg, "Role of antiprogestational therapy for meningiomas," Human Reproduction, vol. 9, supplement 1, pp. 202207, 1994.

[56] K.-P. Lesch, W. Schott, H.-G. Engl, S. Groß, and P. Thierauf, "Gonadal steroid receptors in meningiomas," Journal of Neurology, vol. 234, no. 5, pp. 328-333, 1987. 
[57] D. W. Hsu, J. T. Efird, and E. T. Hedley-Whyte, "Progesterone and estrogen receptors in meningiomas: prognostic considerations," Journal of Neurosurgery, vol. 86, no. 1, pp. 113-120, 1997.

[58] D. S. Baxter, A. Orrego, J. V. Rosenfeld, and T. Mathiesen, "An audit of immunohistochemical marker patterns in meningioma," Journal of Clinical Neuroscience, vol. 21, no. 3, pp. 421426, 2014.

[59] H. Huang, R. Buhl, H. H. Hugo, and H. M. Mehdorn, "Clinical and histological features of multiple meningiomas compared with solitary meningiomas," Neurological Research, vol. 27, no. 3, pp. 324-332, 2005.

[60] P. Kastner, A. Krust, B. Turcotte et al., "Two distinct estrogenregulated promoters generate transcripts encoding the two functionally different human progesterone receptor forms A and B," The EMBO Journal, vol. 9, no. 5, pp. 1603-1614, 1990.

[61] J. K. Richer, B. M. Jacobsen, N. G. Manning, M. G. Abel, D. M. Wolf, and K. B. Horwitz, "Differential gene regulation by the two progesterone receptor isoforms in human breast cancer cells," The Journal of Biological Chemistry, vol. 277, no. 7, pp. 5209-5218, 2002.

[62] B. M. Jacobsen, J. K. Richer, S. A. Schittone, and K. B. Horwitz, "New human breast cancer cells to study progesterone receptor isoform ratio effects and ligand-independent gene regulation," The Journal of Biological Chemistry, vol. 277, no. 31, pp. 2779327800, 2002.

[63] L. A. Helguero, M. Viegas, A. Asaithamby, G. Shyamala, C. Lanari, and A. A. Molinolo, "Progesterone receptor expression in medroxyprogesterone acetate-induced murine mammary carcinomas and response to endocrine treatment," Breast Cancer Research and Treatment, vol. 79, no. 3, pp. 379-390, 2003.

[64] V. Wargon, L. A. Helguero, J. Bolado et al., "Reversal of antiprogestin resistance and progesterone receptor isoform ratio in acquired resistant mammary carcinomas," Breast Cancer Research and Treatment, vol. 116, no. 3, pp. 449-460, 2009.

[65] G. Sequeira, S. I. Vanzulli, P. Rojas et al., "The effectiveness of nano chemotherapeutic particles combined with mifepristone depends on the PR isoform ratio in preclinical models of breast cancer," Oncotarget, vol. 5, no. 10, pp. 3246-3260, 2014.

[66] J. Chen, J. Wang, J. Shao et al., "The unique pharmacological characteristics of mifepristone (RU486): from terminating pregnancy to preventing cancer metastasis," Medicinal Research Reviews, vol. 34, no. 5, pp. 979-1000, 2014.

[67] X. Cui, Z. W. Lazard, P. Zhang, T. A. Hopp, and A. V. Lee, "Progesterone crosstalks with insulin-like growth factor signaling in breast cancer cells via induction of insulin receptor substrate-2," Oncogene, vol. 22, no. 44, pp. 6937-6941, 2003.

[68] D.-Q. Li, Z.-B. Wang, J. Bai et al., "Effects of mifepristone on proliferation of human gastric adenocarcinoma cell line SGC7901 in vitro," World Journal of Gastroenterology, vol. 10, no. 18, pp. 2628-2631, 2004.

[69] A. D. Norden, J. J. Raizer, L. E. Abrey et al., "Phase II trials of erlotinib or gefitinib in patients with recurrent meningioma," Journal of Neuro-Oncology, vol. 96, no. 2, pp. 211-217, 2010.

[70] P. Y. Wen, E. Quant, J. Drappatz, R. Beroukhim, and A. D. Norden, "Medical therapies for meningiomas," Journal of Neuro-Oncology, vol. 99, no. 3, pp. 365-378, 2010.

[71] N. Andrae, E. Kirches, R. Hartig et al., "Sunitinib targets PDGFreceptor and Flt3 and reduces survival and migration of human meningioma cells," European Journal of Cancer, vol. 48, no. 12, pp. 1831-1841, 2012.

[72] M. J. A. Puchner, V. H. Hans, A. Harati, F. Lohmann, M. Glas, and U. Herrlinger, "Bevacizumab-induced regression of anaplastic meningioma," Annals of Oncology, vol. 21, no. 12, pp. 2445-2446, 2010. 


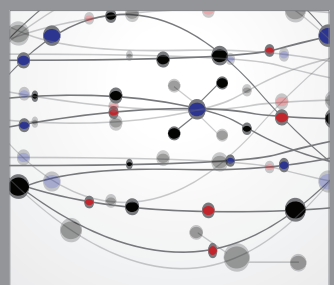

The Scientific World Journal
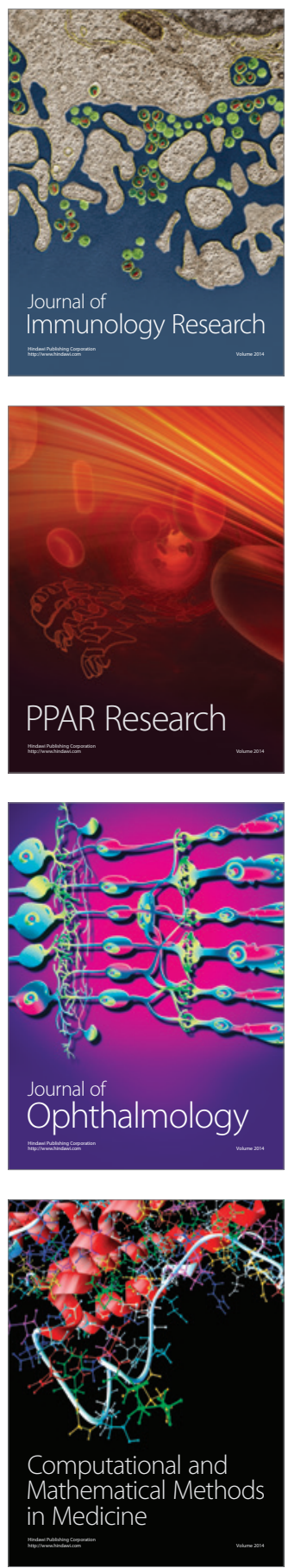

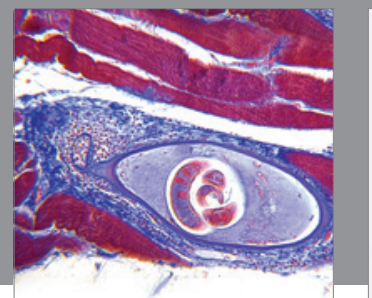

Gastroenterology

Research and Practice
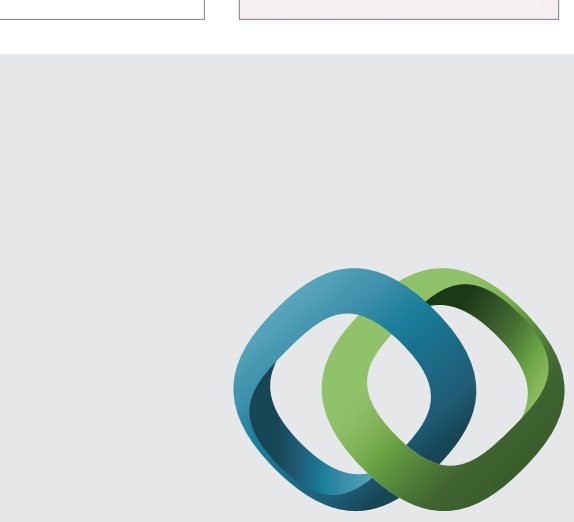

\section{Hindawi}

Submit your manuscripts at

http://www.hindawi.com
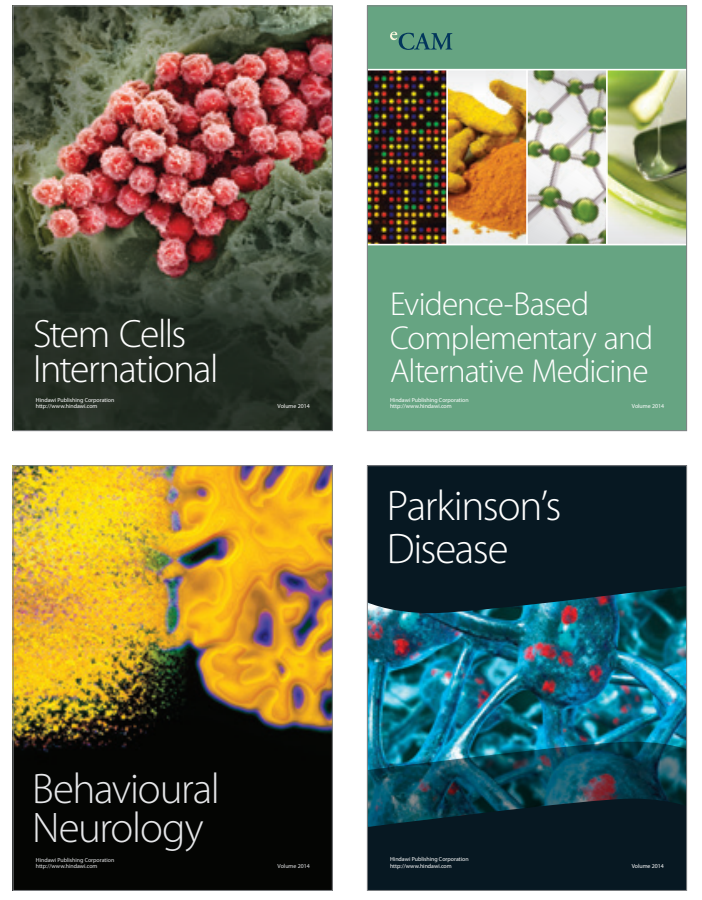
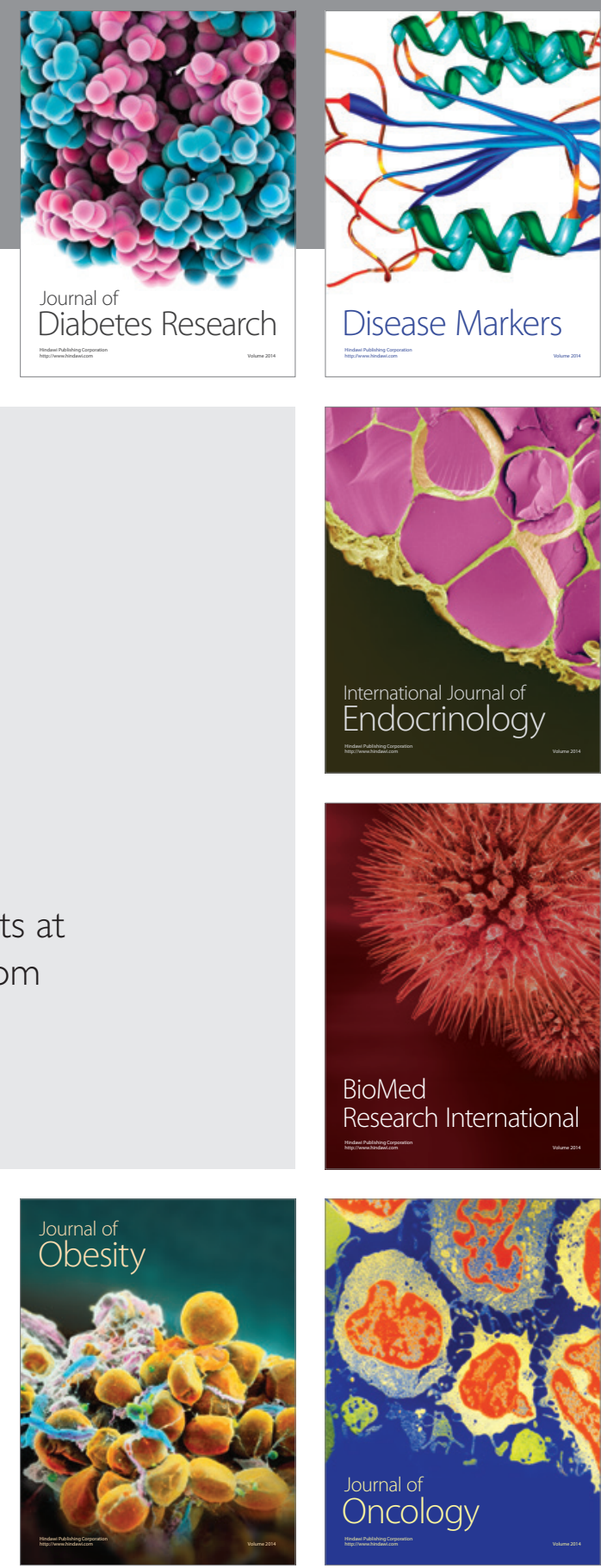

Disease Markers
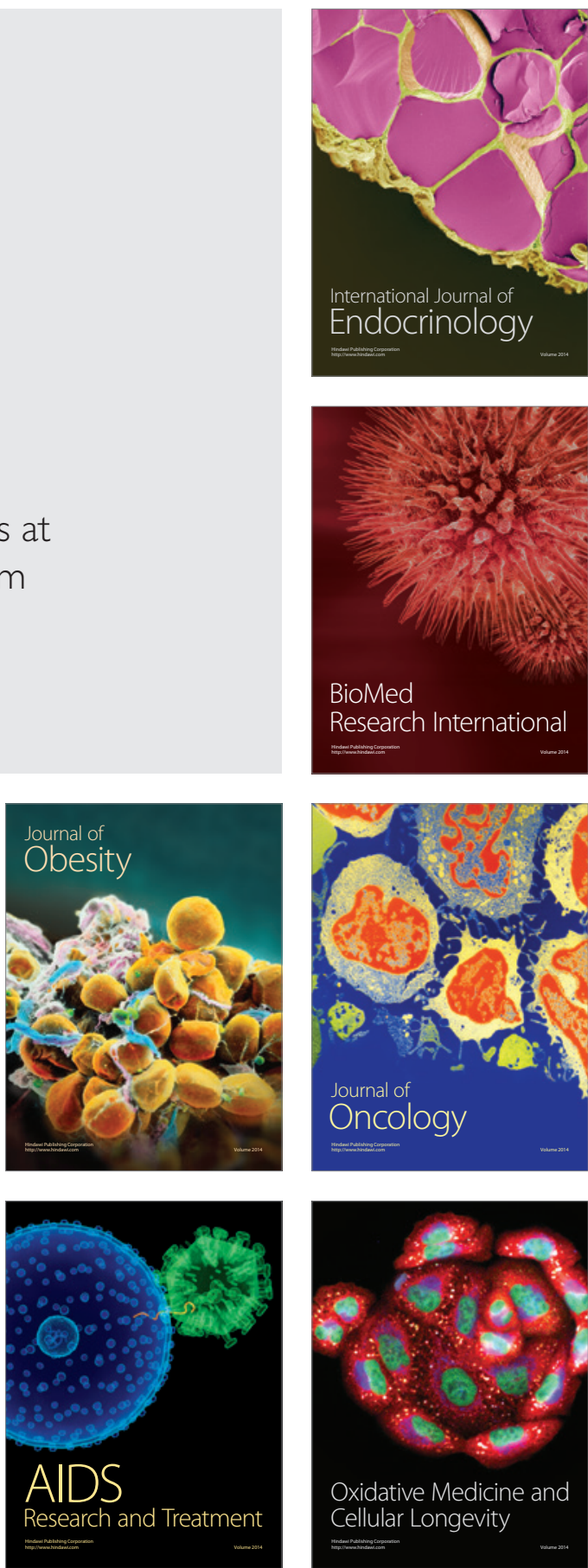